\title{
Taking the Measure of Massive Stars and their Environments with the CHARA Array Long-baseline Interferometer
}

\author{
Douglas R. Gies \\ Center for High Angular Resolution Astronomy, Georgia State University, P.O. Box 5060, \\ Atlanta, Georgia 30302-5060, U.S.A. \\ email: gies@chara.gsu.edu
}

\begin{abstract}
Most massive stars are so distant that their angular diameters are too small for direct resolution. However, the observational situation is now much more favorable, thanks to new opportunities available with optical/IR long-baseline interferometry. The Georgia State University Center for High Angular Resolution Astronomy Array at Mount Wilson Observatory is a six-telescope instrument with a maximum baseline of 330 meters, which is capable of resolving stellar disks with diameters as small as 0.2 milliarcsec. The distant stars are no longer out of range, and many kinds of investigations are possible. Here we summarize a number of studies involving angular diameter measurements and effective temperature estimates for OB stars, binary and multiple stars (including the $\sigma$ Orionis system), and outflows in Luminous Blue Variables. An enlarged visitors program will begin in 2017 that will open many opportunities for new programs in high angular resolution astronomy.
\end{abstract}

\section{The CHARA Array}

Georgia State University operates the Center for High Angular Resolution Astronomy (CHARA) Array at Mount Wilson Observatory, a six-telescope interferometer designed for studies of stars and their environments at milliarcsec (mas) angular scales. The CHARA Array's six 1-m aperture telescopes are arranged in a $Y$-shaped configuration yielding 15 interferometric baselines from 33 to 331 meters as well as 10 independent closure phases. These include the longest OIR baselines yet implemented anywhere in the world and permit resolutions at the sub-mas level. The facility's primary components and sub-systems are described fully by ten Brummelaar et al. (2005), and they include telescopes, evacuated light pipes, optical path length compensation, and beam management. The final step of beam combination is made with a set of different combiners that were built primarily by members of the "CHARA Collaboration" that includes groups (and their PIs) from l'Observatoire de Paris (Vincent Coudé du Foresto), University of Michigan (John Monnier), University of Sydney (Peter Tuthill), the Australian National University (Michael Ireland), l'Observatoire de la Côte d'Azur (Denis Mourard), and the University of Exeter (Stefan Kraus). These combiners are optimized for different wavelength bands and numbers of telescopes (Table 1).

The Array is primarily used to measure interferometric visibility, a quantity that is directly related to the Fourier transform of the spatial intensity of an object in the sky. Measurements at lower spatial frequencies are related to the angular diameter of a star (and its shape if measurements are available with multiple telescope pairs), while higher spatial frequencies provide important diagnostics of the distribution of spatial intensity (for use in measuring limb darkening and spot distributions, for example). In addition, 
Table 1. CHARA Array Beam Combiners

\begin{tabular}{|c|c|c|c|c|c|c|}
\hline Combiner & \# Tel. & Band & $\begin{array}{l}\text { Typ. } \\
\text { Limit. } \\
\text { Mag. }\end{array}$ & $\begin{array}{l}\text { Best } \\
\text { Perf. } \\
\text { Mag. }\end{array}$ & $\begin{array}{c}\text { Spectral } \\
\text { Resol. } \\
\text { Power }\end{array}$ & Reference \\
\hline \multicolumn{7}{|c|}{ Currently Operating } \\
\hline CLASSIC & 2 & $H$ or $K$ band & 7.0 & 8.5 & Broad band & ten Brummelaar et al. (2005) \\
\hline CLIMB & 3 & $H$ or $K$ band & 6.0 & 7.0 & Broad band & ten Brummelaar et al. (2013) \\
\hline JouFLUOR & 2 & $K$ & 3.5 & 5.0 & Broad band & Scott et al. $(2013)$ \\
\hline $\begin{array}{l}\text { VEGA } \\
\text { (hi-res) }\end{array}$ & 2 or 3 & $\begin{array}{c}2 \times 7 \mathrm{~nm}, \\
\text { in } 480-850 \mathrm{~nm}\end{array}$ & 4.0 & 5.0 & $\begin{array}{l}30000 \\
30000\end{array}$ & Mourard et al. (2011) \\
\hline $\begin{array}{l}\text { VEGA } \\
\text { (med-res) }\end{array}$ & 2 or 3 & $\begin{array}{c}2 \times 35 \mathrm{~nm} \\
\text { in } 480-850 \mathrm{~nm}\end{array}$ & 6.5 & 7.5 & $\begin{array}{l}6000 \\
6000\end{array}$ & Mourard et al. (2011) \\
\hline MIRC & 6 & $H$ & 4.0 & 6.0 & 42 & Monnier et al. (2006) \\
\hline PAVO & 2 & $630-900 \mathrm{~nm}$ & 7.0 & 8.0 & 30 & Ireland et al. (2008) \\
\hline \multicolumn{7}{|c|}{ Planned } \\
\hline MIRCX & 6 & $J+H$ & 7.0 & 9.0 & 42 & Kraus et al. (2016) \\
\hline MYSTIC & 6 & K & 7.5 & 8.5 & 42 & Monnier et al. (2016) \\
\hline SPICA & 6 & $550-850 \mathrm{~nm}$ & 8.9 & 11 & 100 & Mourard (2016) \\
\hline
\end{tabular}

closure phase measurements from three or more telescopes are related to asymmetries in the spatial distribution and are critical in image reconstruction from interferometry. I show in Figure 1 the range in possible measurements for the Classic two-telescope beam combiner. The $x$-axis shows the angular diameter of an object in the sky (measured in milli-arcseconds) and the $y$-axis shows the magnitude of the object in the near-infrared $H$-band (in the absence of interstellar extinction). The dark, solid lines show how the angular diameters vary with magnitude for stellar effective temperatures of $T_{\text {eff }}=3500$, 7000 , and $25000 \mathrm{~K}$. Hot objects have much larger surface intensities than cooler ones, so that the hotter O- and B-type stars appear smaller in the sky than cooler stars do at a given apparent magnitude. The gray diagonal lines show the apparent semimajor axis of binary stars of total mass $0.9,2.3$, and $34 M_{\odot}$ for M0 V, G0 V, and B0 V pairs, respectively, over a range in distance. Interferometers like the CHARA Array are able to resolve and map astrometric orbits for all kinds of close, spectroscopic binaries. The representative sizes of several kinds of objects that host gaseous disks are also shown in Figure 1. I discuss some of the remarkable observational results on massive stars from the CHARA Array in the next two sections.

\section{Resolving massive star photospheres and outflows}

The CHARA Array provides access to stars all along the main sequence. A measurement of the angular diameter together with a parallax yields the physical radius, and the effective temperature is derived from the angular diameter and spectral energy distribution. Thus, CHARA measurements lead directly to an observational Hertzsprung-Russell diagram that provides a test for theoretical models. Work has steadily progressed from cooler stars (Boyajian et al. 2015), through the A-type stars (Jones et al. 2015), and into the B-star domain (Maestro et al. 2013). Kathryn Gordon (GSU) is currently investigating the angular diameters of more massive stars in a sample that includes six O-type stars. Many of the early-type stars are rapid rotators, and the first science paper from CHARA highlighted the rotationally distorted shape of the B-star Regulus (McAlister et al. 2005). The CHARA/MIRC combiner has been used in multi-telescope 


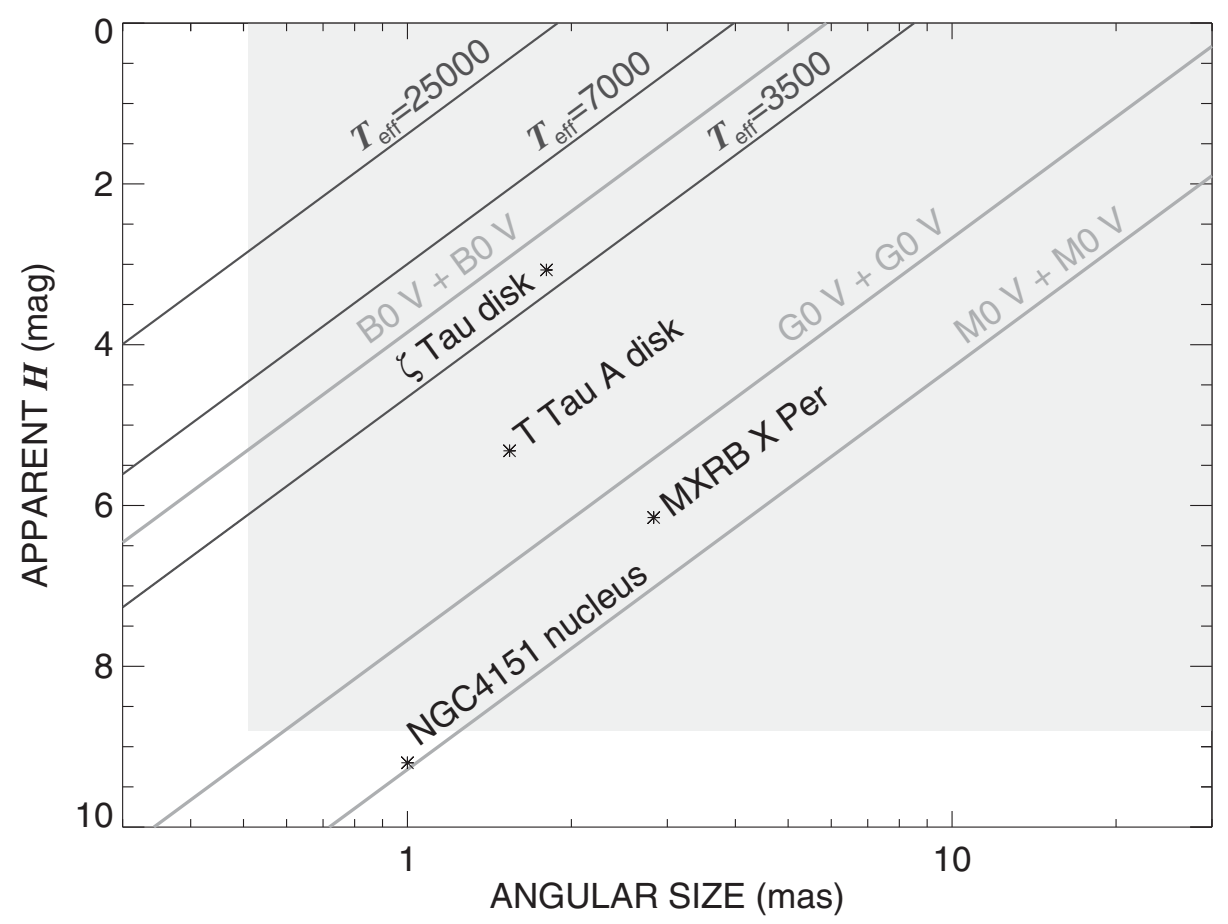

Figure 1. The approximate range in size and magnitude of targets accessible to CHARA Classic in $H$-band. Dark lines show diameters of stars of different temperature and gray lines show the semi-major axis of a 10 day binary over a range in distance, and these may be translated horizontally according to $(P / 10)^{2 / 3}$. Note that the magnitude limit given here is conservative, and it is possible to go fainter in excellent seeing conditions.

configurations to obtain the first images of rapid rotators that clearly show the the hotter poles and cooler equators that result from gravity darkening (Monnier et al. 2007; Zhao et al. 2009; Che et al. 2011). Jones et al. (2016) modeled Array observations of the low inclination, rapid rotator, $\kappa$ And that is the A0 V, host star of a directly imaged, low-mass planetary companion.

Massive O- and B-type stars evolve to become Cepheids, red supergiants, and luminous blue variable (LBV) stars, and investigations are underway of the properties of these older objects. Mérand et al. (2006) discovered extended circumstellar envelopes surrounding the Cepheids Polaris and $\delta$ Cep using very accurate visibility measurements from CHARA/FLUOR. These halos have radii several times larger than the star and contribute a few percent of the $K$-band flux. Additional observations by Mérand et al. (2007) indicate that circumstellar envelopes are often found around pulsating Cepheids, but are absent among similar non-pulsating supergiants. This indicates that the halos may be the result of pulsation-driven mass loss. Gallenne et al. (2012) measured diameters, distances, and pulsation modes in the classical Cepheids FF Aql and T Vul. Gallenne et al. $(2013,2015)$ detected the faint orbiting companions of a number of Cepheids enabling the determination of their relative brightness, orbital elements, precise and independent distance estimates, and masses. Combined with GAIA distances, these measurements are required to reduce eventually the systematic error of the Cepheid-based extragalactic distance scale.

The CHARA Array is ideal for studying convective structures in red supergiants, and Baron et al. (2014) presented some of the first results from CHARA/MIRC in the 
$H$-band. The reconstructed images of two red supergiants show some evidence for the predicted large scale cells, and Ryan Norris (GSU) is now studying an enlarged sample. Richardson et al. (2013) used CHARA/MIRC to observe the supergiant P Cygni, one of the prototypes of the class of LBVs. Their reconstructed image of P Cygni clearly shows the spherical halo of flux created by the star's dense stellar wind. Richardson et al. (2016) found that the candidate LBV star MWC 314 is an interacting binary undergoing massive Roche lobe overflow that completely envelopes the mass gainer, perhaps what $\beta$ Lyr experienced at an earlier epoch.

\section{Massive binary stars}

Angular orbits from CHARA Array data provide masses and distances for close and wide binaries with orbital periods from days to centuries. For example, Schaefer et al. (2016) determined an astrometric orbit for the $\sigma$ Ori Aa,Ab binary that they combined with the spectroscopic orbit to yield very accurate masses and distance to this Ori OB1 association member (with an uncertainty of only $0.3 \%$ ). Zhao et al. (2008) imaged the famous interacting binary $\beta$ Lyr at four epochs covering approximately half the 12.9-day orbit. Those images clearly reveal the bright and elongated mass donor and thick disk surrounding the mass gainer comprising $\beta$ Lyr. This represents the first direct detection of the flux of the gas torus obscuring the mass gainer star, after a century of attempts to detect the gainer by spectroscopy. The extreme mass transfer rates that occur near minimum separation in an interacting binary may lead to the formation of a circumbinary disk that is fed through leaky gas outflows through the outer axial Lagrangian points. Such mass loss processes may produce a dense and opaque circumbinary disk such as that observed in the $\epsilon$ Aur system. This enigmatic 27.1-yr eclipsing system was the subject of an observing campaign at CHARA that spanned the recent eclipse. Kloppenborg et al. (2010, 2015) presented images of the eclipse event made from CHARA/MIRC showing the silhouette of the optically-thick disk passing in front of the F-type supergiant primary.

The mass gainer in an interacting binary may be spun up to near the critical rate, and some of the rapidly rotating Be stars are probably the result of binary mass transfer. The former mass donor star may be stripped of its envelope, and its core will appear as a faint and hot subdwarf star (Peters et al. 2016). The companion of the Be star $\phi$ Per is one such case. Mourard et al. (2015) detected and mapped the orbit of the faint companion, and their CHARA/VEGA observations of the Be disk show that the gas moves in the same sense as the orbit of the companion. This finding supports the hypothesis that the Be star was spun up by mass and angular momentum transfer, so that the angular momentum being lost into the Be disk reflects the original orbital angular momentum of the mass donor. These remarkable results speak eloquently of the power interferometric imaging possesses in studying such classical systems. Binary stars continue to be a touchstone for astrophysics, renewed with each major advance in measurement technology.

\section{Open access to CHARA}

CHARA recently received funding from the U.S. National Science Foundation MidScale Innovations Program to (1) provide open access to the community for 50 to 75 nights per year, and (2) provide an on-line data pipeline and archive of CHARA observations. Time will be allocated through the U.S. National Optical Astronomy Observatory Time Allocation Committee review beginning in 2017B (August to Dec 2017). Plans are underway for community workshops to help guest observers develop new programs and to acquire the knowledge needed to obtain observations and reduce and analyze the 
data. We anticipate that the new open access program will bring exciting new ideas for observational work and will help make optical long baseline interferometry part of the basic toolbox for investigations in stellar astrophysics.

\section{Acknowledgements}

This material is based upon work supported by the National Science Foundation under Grants No. AST-1211929 (McAlister), AST-1411654 (Gies), and AST-1636624 (ten Brummelaar).

\section{References}

Baron, F., Monnier, J. D., Kiss, L. L., et al. 2014, ApJ, 785, 46

Boyajian, T., von Braun, K., Feiden, G. A., et al. 2015, MNRAS, 447, 846

Che, X., Monnier, J. D., Zhao, M., et al. 2011, ApJ, 732, 68

Gallenne, A., Mérand, A., Kervella, P., et al. 2015, A\&\&A, 579, A68

Gallenne, A., Mérand, A., Kervella, P., et al. 2016, MNRAS, 461, 1451

Gallenne, A., Monnier, J. D., Mérand, A., et al. 2013, A\&BA, 552, A21

Ireland, M. J., Mérand, A., ten Brummelaar, T. A., et al. 2008, Proc. SPIE, 7013, 701324

Jones, J., White, R. J., Boyajian, T., et al. 2015, ApJ, 813, 58

Jones, J., White, R. J., Quinn, S., et al. 2016, ApJ (Letters), 822, L3

Kloppenborg, B., Stencel, R., Monnier, J. D., et al. 2010, Nature, 464, 870

Kloppenborg, B., Stencel, R., Monnier, J. D., et al. 2015, ApJS, 220, 14

Kraus, S., Anugu, N., Davies, C., \& Monnier, J., 2016, CHARA Meeting, http://www.chara.gsu.edu/files/2016Meeting/kraus.MIRCx.pdf

Maestro, V., Che, X., Huber, D., et al. 2013, MNRAS, 434, 1321

McAlister, H. A., ten Brummelaar, T. A., Gies, D. R., et al. 2005, ApJ, 628, 439

Mérand, A., Aufdenberg, J. P., Kervella, P., et al. 2007, ApJ, 664, 1093

Mérand, A., Kervella, P., Coudé du Foresto, V., et al. 2006, A\&A, 453, 155

Monnier, J., Le Bouquin, J.-B., Jocou, L., et al. 2016, CHARA Meeting, http://www.chara.gsu.edu/files/2016Meeting/Monnier_MYSTIC_public_1.pdf

Monnier, J. D., Pedretti, E., Thureau, N., et al. 2006, Proc. SPIE, 6268, 62681P

Monnier, J. D., Zhao, M., Pedretti, E., et al. 2007, Science, 317, 342

Mourard, D. 2016, CHARA Meeting, http://www.chara.gsu.edu/files/2016Meeting/Mourard.pdf

Mourard, D., Bério, P., Perraut, K., et al. 2011, A\&SA, 531, A110

Mourard, D., Monnier, J. D., Meilland, A., et al. 2015, A\&A, 577, A51

Peters, G. J., Wang, L., Gies, D. R., \& Grundstrom, E. D. 2016, ApJ, 828, 47

Richardson, N. D., Moffat, A. F. J., Maltais-Tariant, R., et al. 2016, MNRAS, 455, 244

Richardson, N. D., Schaefer, G. H., Gies, D. R., et al. 2013, ApJ, 769, 118

Schaefer, G. H., Hummel, C. A., Gies, D. R., et al. 2016, AJ, 152, 213

Scott, N. J., Millan-Gabet, R., Lhomé, E., et al. 2013, Journal of Astronomical Instrumentation, 2, 40005

ten Brummelaar, T. A., McAlister, H. A., Ridgway, S. T., et al. 2005, ApJ, 628, 453

ten Brummelaar, T. A., Sturmann, J., Ridgway, S. T., et al. 2013, Journal of Astronomical Instrumentation, 2, 40004

Zhao, M., Gies, D., Monnier, J. D., et al. 2008, ApJ (Letters), 684, L95

Zhao, M., Monnier, J. D., Pedretti, E., et al. 2009, ApJ, 701, 209 